\title{
Mineralogical and geochemical characterization of the Benavila (Portugal) bentonites
}

\author{
DR. JAVIER GARCÍA-RIVAS ${ }^{1,2}$, MARIA ISABEL DIAS ${ }^{2}$, \\ EMILIA GARCÍA ROMERO ${ }^{3,4}$, MERCEDES SUÁREZ \\ BARRIOS $^{1}$ AND ISABEL PAIVA ${ }^{2}$ \\ ${ }^{1}$ University of Salamanca \\ ${ }^{2}$ IST / University of Lisbon \\ ${ }^{3}$ Complutense University of Madrid \\ ${ }^{4}$ Institute of Geosciences (IGEO) (Complutense University of \\ Madrid - Consejo Superior de Investigaciones Científicas) \\ Presenting Author: javiergr_89@usal.es
}

Bentonites are clayey industrial rocks of great economic interest whose main component are smectites. These minerals are phyllosilicates with physical-chemical properties of key importance for their industrial application, such as high specific surfaces, high cation exchange capacity (CEC) and low layer charge, which allows them to react with inorganic and organic reagents, being adsorbed into their structure. In addition, their swelling and rheological properties and high plasticity also play a very important role.

The Benavila (Portugal) bentonite deposit is the largest in continental Portugal and was formed by the weathering of granodiorites and quartzdiorites. In this work, 9 samples from a representative outcrop of this deposit were characterized in terms of mineralogy (XRD and TEM-AEM) and geochemistry (ICPMS). Samples were studied in the bulk rock fraction as well as in the fractions below 63,38 and $2 \mu \mathrm{m}$.

The mineralogical characterization through XRD shows that all samples are mainly constituted by dioctahedral smectites, along with other minerals such as carbonates, tectosilicates, amphiboles and other clay minerals. The TEM-AEM analysis shows that all these dioctahedral smectites are classified as ferrian montmorillonitic beidellites always, although with variable interlayer charges.

The geochemical analysis allows to observe a certain depletion in REE and other heavy elements in the samples with higher content in smectites. However, the most important fact observed is not the depletion or enrichment on certain elements, but the calculation of different weathering indexes, such as the CIA. This index shows a very interesting decrease of the weathering following a certain path, which matches with the presence of amphiboles (interpreted as relict and non-weathered minerals from the plutonic parent rock) and an increasing interlayer charge of the smectites.

It is, therefore, possible to conclude that a lower weathering of the parent rock results in higher interlayer charges of the neoformed smectites.

Acknowledgements: Financial support of the SA0107P20 (Consejería de Educación, Junta de Castilla y León, Spain), PID2019-106504RB-I00 (Ministerio de Ciencia e Innovación, Spain) and UID/Multi/04349/2019 (FCT, Portugal) projects is acknowledged. Javier García-Rivas thanks the FCT for postdoctoral fellowship BL233/2019_IST-ID at the C2TN. 